Théologiques

Théologiques

\title{
Ce que devenir juif veut dire
}

\section{Sébastien Tank-Storper}

Volume 21, numéro 2, 2013

\section{La conversion}

URI : https://id.erudit.org/iderudit/1028466ar

DOI : https://doi.org/10.7202/1028466ar

Aller au sommaire du numéro

Éditeur(s)

Faculté de théologie et de sciences des religions, Université de Montréal

ISSN

1188-7109 (imprimé)

1492-1413 (numérique)

Découvrir la revue

\section{Citer cet article}

Tank-Storper, S. (2013). Ce que devenir juif veut dire. Théologiques, 21(2), 159-178. https://doi.org/10.7202/1028466ar

\section{Résumé de l'article}

En affirmant que devenir juif, c'est devenir un juif pratiquant et non un juif " ethnique » ou " national », et en tentant de s'arroger le monopole sur les conversions au judaïsme, notamment en Israël, les institutions religieuses de sensibilité orthodoxe défendent l'idée qu'elles se font de ce qu'être juif veut dire : un collectif soumis à la loi. Elles entendent ainsi lutter contre ce qu'elles considèrent être une réification de l'identité juive dans une entité nationale israélienne ou contre sa réduction à un groupe ethnique ou à une identité dont l'héritage ne constituerait plus un principe structurant mais se réduirait à quelques traits discriminants.
Ce document est protégé par la loi sur le droit d'auteur. L'utilisation des services d'Érudit (y compris la reproduction) est assujettie à sa politique d'utilisation que vous pouvez consulter en ligne.

https://apropos.erudit.org/fr/usagers/politique-dutilisation/ 


\title{
Ce que devenir juif veut dire
}

\author{
Sébastien TANK-STORPER" \\ Chargé de recherche au CNRS \\ École des Hautes Études en sciences sociales (Paris, France)
}

La notion de frontière ethnique élaborée par Fredrik Barth a été déterminante pour la compréhension des groupes ethniques.

Le caractère novateur de la notion d'ethnic boundary, attesté par l'exceptionnelle influence de son auteur [Fredrik Barth, ndla], tient à l'idée que ce sont en réalité ces frontières ethniques, et non pas le contenu culturel interne, qui définissent le groupe ethnique et permettent de rendre compte de sa persistance. [...] L'élucidation des phénomènes d'ethnicité passe alors par l'analyse générative des conditions d'établissement, de maintien, de transformation des frontières entre les groupes. (Poutignat et Streiff-Fenart 1995, 166-167)

Dans cette perspective, ce qui constitue un danger pour tout groupe ethnique en situation de contact avec d'autres groupes n'est pas tant la perte de certains de ses traits culturels que le brouillage de ses frontières. Pour rester pertinente, l'identité ethnique doit ainsi être enserrée dans un ensemble d'interactions sociales garantissant que les individus demeurent soit à l'intérieur, soit à l'extérieur du groupe, limitant au maximum les statuts intermédiaires. Ces dispositifs peuvent s'appuyer aussi bien sur la mise en avant de traits culturels discriminants (mais pas nécessairement sur l'ensemble des traits culturels manifestes) que sur des procédures

* Sébastien Tank-Storper est chargé de recherche au CNRS et membre du CEIFR (Centre d'études interdisciplinaires des faits religieux) de l'École des Hautes Études en sciences sociales à Paris. Son travail porte sur les questions liées au pluralisme religieux au sein du judaïsme contemporain et interroge notamment les processus politiques de construction de l'orthodoxie et de l'hétérodoxie religieuses. Ses recherches l'ont également conduit à Buenos Aires, en Argentine, où il s'intéresse aux conséquences de l'attentat de 1994 contre l'édifice de la AMIA, la principale institution juive d'Argentine. Il a publié (2007) Juifs d'élection. Se convertir au judaïsme, Paris, CNRS éditions, et, avec Olivier Bobineau, (2012) [2007¹] Sociologie des religions, Paris, Armand Colin. 
d'encadrement et d'explicitation des règles d'appartenance et de contrôle des logiques matrimoniales.

Les catégories ethniques formeraient ainsi « une coquille organisationnelle à l'intérieur de laquelle peuvent être mis des contenus de formes et dimensions variées dans des systèmes socio-culturels différents. " (Barth 1995, 211-212) Les contenus de l'identité (linguistiques, économiques, culturels, religieux, etc.) sont souvent manipulés pour pérenniser ou renforcer les frontières du groupe. Certains traits jugés non pertinents dans une situation d'interaction donnée peuvent être abandonnés tandis que d'autres jusque-là périphériques peuvent être surdéterminés pour répondre à une interaction sociale inédite. Barth a ainsi permis de rompre avec des approches essentialistes des identités et des groupes ethniques, qui ne peuvent plus dans sa logique être pensés selon des traits culturels irréductibles. Les groupes ethniques ne devant plus être définis par leurs traits culturels ou religieux, mais par la manière dont ils définissent et entretiennent la frontière qui les sépare des autres groupes.

Pour autant, la désubstantialisation des identités minoritaires n'est pas sans poser problème quand elle est appliquée aux groupes dits "ethnoreligieux ", comme l'est notamment souvent qualifié le judaïsme qui nous occupera dans le cours de ce texte. La relégation des contenus identitaires, et notamment des contenus religieux, à des variables mobilisées et instrumentalisées pour (re)modeler les frontières du groupe dans ses interactions avec les autres groupes, conduit à refuser toute consistance aux faits religieux, devenus, pour reprendre une terminologie marxiste, de simples phénomènes superstructurels de faits ethniques infrastructurels. Le fait religieux serait un fait sans consistance, socialement non pertinent et sans réelle capacité à produire des effets sociaux. Il n'aurait pas de rationalité propre et serait soumis à des raisons qui lui sont extérieures.

Les travaux désormais classiques de Max Weber sur la capacité des éthiques et des représentations religieuses à produire des effets sociaux invitent pourtant à discuter ce type d'approche. L'auteur de L'Éthique protestante et l'esprit du capitalisme montre ainsi que les systèmes religieux sont à même de produire des "éthiques de conviction" au nom desquelles les acteurs sociaux agissent en dehors de tout autre cadre de rationalité. Serait-ce donc que, quand ils sont enchâssés dans une identité de type ethnique, les systèmes religieux perdraient toute capacité à orienter les actions individuelles? 
Il ne s'agira pas ici de débattre si le judaïsme est prioritairement ethnique ou prioritairement religieux. Il ne s'agira pas non plus de remettre en cause les acquis d'une compréhension des groupes ethniques ou ethnoreligieux comme des groupes en négociation constante de leur identité en fonction du contexte et de leurs interactions avec d'autres groupes sociaux, au profit d'une resubstantialisation des identités ethniques ou religieuses. Le judaïsme notamment, qui constitue le terrain à partir duquel nous tentons de nous saisir de la question, ne peut être saisi par le prisme exclusif de ses contenus. La grande plasticité de ses formes à travers le temps et l'espace démontre à quel point le fait juif ne peut se réduire à quelque trait irréductible que ce soit. Chaque rupture politique ou historique a donné lieu, au long de son histoire, à des processus de réévaluation parfois massifs de ses formes d'organisation politique et de ses formulations religieuses, avec pour objectif explicite de permettre la pérennité du groupe.

Mon propos sera plutôt d'essayer de montrer que les conflits contemporains autour de la définition des frontières du judaïsme - conflits qui se cristallisent notamment autour du débat sur les conversions visant à définir à quelles conditions un individu qui n'est pas né juif selon les critères fixés par la Halakha (la loi juive) peut être admis au sein de l'Alliance - ne peuvent se comprendre par le prisme exclusif de l'ethnicité et qu'ils doivent être également saisis dans le cadre «d'éthiques de la conviction» (pour reprendre une terminologie wébérienne) qui s'enracinent dans des rationalités de type religieux. Il s'agira, en d'autres termes, de tempérer l'idée que les juifs (ou tout autre groupe ethnique ou ethno-religieux) ne se définissent comme juifs que dans leur rapport aux autres, mais qu'ils le sont aussi pour eux-mêmes et en fonction de l'idée qu'ils se font de ce qu'est et de ce que signifie être juif. À l'identité/altérité que propose la perspective de Fredrik Barth, il faudrait ainsi ajouter (mais sans pour autant substituer) l'idée d'une identité/ipséité telle que la propose Paul Ricœur dans son livre Soi-même comme un autre (1990): une identité réflexive fondée ni sur la seule confrontation signifiante avec l'altérité, ni sur la seule persistance d'un caractère dans le temps et dans l'espace (et qui conduit à résumer l'identité à des traits immuables), mais aussi sur la volonté de rester le plus fidèle possible à une promesse, à l'idée que l'on se fait de soi-même.

Cette proposition peut conduire à repenser la question des contenus culturels et de leurs effets sociaux. Si le chercheur doit se garder d'une vision essentialiste des identités ethniques ou des religions en privilégiant une approche constructiviste des logiques identitaires et religieuses, il doit cependant rester attentif aux logiques des acteurs qui travaillent activement 
à la définition des frontières et qui, parfois, agissent au nom de conceptions essentialistes de l'identité. C'est aussi en allant au plus près de la logique des différents acteurs que l'on peut rendre aux phénomènes d'identité toute leur complexité et en saisir tous les enjeux.

L'objet de ce texte sera ainsi de montrer, à partir de la situation sociale particulière que tissent les demandes de conversion adressées aux institutions juives contemporaines, que les principaux enjeux liés à la définition des frontières du judaïsme sont non seulement de définir les contenus légitimes de l'identité juive, mais aussi peut-être surtout de définir la nature du lien qui unit les juifs: une communauté d'origine (définition ethnique de l'identité juive)? Une communauté de destin (définition nationale de l'identité juive)? Une communauté de croyance et de pratiques (définition religieuse de l'identité juive) ? Que signifie, en d'autres mots, devenir juif? Est-ce s'agréger à un Peuple, embrasser son destin ? Est-ce intégrer un Étatnation, Israël, avec ses lois, ses institutions politiques? Est-ce embrasser un mode de vie ou une culture? Est-ce adhérer à un système de croyances, à une vision du monde?

Nous partirons ainsi des trajectoires d'identification des convertis, afin d'en comprendre les principaux ressorts. Si les parcours qui mènent des individus à vouloir se convertir au judaïsme sont multiples, ils s'inscrivent dans leur très grande majorité dans un projet de transmission formelle de l'identité juive. Il s'agit, en somme, d'être en mesure d'engendrer des enfants juifs et de les élever au sein d'un foyer juif, sans pour autant que le caractère juif de ce foyer soit pensé en termes religieux. De leur côté, les rabbins orthodoxes auxquels les candidats sont plus ou moins contraints de s'adresser pour se convertir entendent imposer, à travers les critères posés à l'acceptations des candidats, une conception religieuse de l'identité, fondée sur l'observance des mitsvot (de la loi juive), au risque de brouiller les frontières de l'identité juive en fermant la porte des conversions à des individus qui, par ailleurs, s'identifient comme juifs et participent activement à certains modes de sociabilité juive.

\section{Se convertir}

Quand je le rencontre pour la première fois en 1998, Olivier poursuit un doctorat de physique tout en effectuant un service civil. Même s'il a été baptisé à sa naissance, ses parents lui ont donné une éducation plutôt laïque. Son père avait rompu avec le catholicisme et ne dispensa aucune instruction religieuse à ses enfants. 
Olivier rencontre le judaïsme pour la première fois à l'âge de sept ou huit ans à travers de vieux numéros d'Historia consacrés à la Shoah - la destruction des juifs d'Europe durant la Seconde Guerre mondiale - que son grand-père conservait dans sa maison de campagne. "C'est le premier contact que j'ai eu avec le monde juif. À travers la Shoah.» La découverte de cet événement bouleverse l'enfant au point que cela deviendra pour lui une véritable obsession.

Adolescent, il se dit fasciné par Israël. Il ne supporte pas les blagues sur les juifs et il lui arrive même de se battre pour ça. La fascination qu'il dit éprouver pour le judaïsme, «une fascination sans rien en connaître hormis les souffrances ", ne l'empêche cependant pas de s'intéresser, à cette époque, de très près au bouddhisme. Il lit de nombreux textes sur ce sujet, mais son intérêt reste avant tout intellectuel.

Sa véritable rencontre avec le judaïsme se fait à travers la lecture du livre de Marc-Alain Ouaknin Le Livre brulé, un texte consacré au rapport que le judaïsme entretient avec le livre et avec l'interprétation: "C'était un gros choc. J'ai découvert toute l'herméneutique. Mais ça restait de la curiosité intellectuelle.»

À la fin de son DEA1, il se sépare de son amie et décide de partir en Israël. Il se promène dans le pays, rencontre de nombreuses personnes et commence à s'imprégner de la culture juive. À son retour en France, quelque chose se passe: "petit à petit émerge en moi une foi». Il entreprend d'apprendre l'hébreu. Seul. Il commence à lire la Bible et certains passages du Talmud, toujours seul, s'intéresse à la culture yiddish, dont il apprend la langue, et approfondit ses lectures sur le judaïsme (à travers la lecture de l'œuvre du philosophe Émmanuel Lévinas notamment).

Une année plus tard, il écrit à un ami qu'il avait rencontré en Israël (un converti qui se préparait à devenir rabbin) et lui dit que lui aussi voulait se convertir. "C'était une évidence qui s'imposait à moi. La vision du monde que j'avais s'intégrait complètement dans la vision juive du monde [...]. Pour répondre aux questions spirituelles qui me hantent depuis assez longtemps.»

En décembre 1996, il envoie la première lettre au Consistoire, qui ne l'encourage pas à poursuivre dans la voie de la conversion. À la même époque, il rencontre lors d'une conférence sur le judaïsme un jeune juif qui l'invite à venir fêter Shabbat dans sa famille. Il y découvre toute la partie rituelle et festive du judaïsme. Jusqu'alors, la seule pratique qu'il avait adoptée était

1. Diplôme d'Études Approfondies, qui correspond aujourd'hui au Master. 
de ne plus manger de porc. Les paroles, les gestes de la tradition lui étaient inconnus. Mais avant tout, il trouve dans cette famille une jeune femme dont il tombe amoureux et avec qui il projette rapidement de se marier ${ }^{2}$.

À l'image du parcours d'Olivier, et à celle d'une identité juive largement pluralisée, les trajectoires individuelles de conversion au judaïsme témoignent de logiques d'identification qui articulent plusieurs dimensions : mémorielle (avec l'identification aux persécutions juives et notamment à la Shoah), culturelle, éthique, politique, familiale, etc., mais aussi nationale, avec, selon les chiffres disponibles en 2014, environ 3500 conversions validées chaque année par la Rabbanout officielle israélienne et 800 réalisées par l'armée israélienne (Stern 2013). La dimension proprement religieuse est rarement première et jamais exclusive. C'est même rarement "pour la Torah" que se présentent les candidats à la conversion, dont la démarche vise le plus souvent l'intégration dans une communauté perçue davantage comme un peuple, une ethnicité ou une communauté nationale que comme une communauté croyante.

Cela est particulièrement saillant dans le cas des conversions dans le cadre d'un mariage mixte, qui représentent entre 80 et $90 \%$ des cas de conversions réalisées en diaspora et qui concernent presque toujours les femmes ${ }^{3}$. Le mariage mixte, quand il donne lieu à la conversion des épouses, témoigne en effet de la part des époux d'un rapport ambivalent au judaïsme et à la judéité. Il révèle la distance que prennent ces hommes juifs vis-à-vis des normes de l'identité juive, et notamment de la règle d'endogamie, distance qui n'exclut cependant pas d'autres formes d'attachement à l'identité juive, comme à l'État d'Israël, à la culture yiddish ou judéo-arabe, ou encore à une culture familiale fortement façonnée par la tradition juive. Mais entre endogamie juive et homogamie sociale, ils font le choix de l'homogamie ou, pour le dire de manière moins abrupte, ils privilégient la conception romantique du mariage à l'injonction à se marier au sein du groupe. Le judaïsme n'est donc pour eux pas vécu comme un

2. Entretien mené par l'auteur en mai 1998 à Paris.

3. Il n'existe pas de statistiques portant sur l'ensemble de la population des convertis au judaïsme. On dispose cependant d'un certain nombre de données quantitatives réalisées par des chercheurs ou par des institutions juives à partir d'échantillons locaux, qui convergent toutes autour d'un pourcentage compris entre 80 et $90 \%$ des cas de conversion réalisés dans le cadre d'un mariage mixte (que ce mariage soit projeté ou déjà contracté). Voir notamment Mayer (1977), Forster et Tabachnik (1991), Montgomery (1991), Levin (1995), Allouche-Benayoun (1997), Barack Fishman (1999). 
système de normes, mais comme une identification qui n'implique pas nécessairement de vivre selon ses principes.

Pour autant, la transgression de la règle d'endogamie peut être vécue comme problématique, voire comme une trahison vis-à-vis de la lignée, notamment en regard de l'identité des enfants. Les juifs aiment à répéter la formule en forme de boutade inversant les termes classiques de l'identité: est juif celui dont les enfants sont juifs, en lieu et place de la règle voulant que soit juif celui dont la mère est juive. La conversion de l'épouse non juive vient alors réparer la transgression et permet la transmission de la judéité aux enfants (puisque c'est le statut de la mère qui détermine celui des enfants).

Le principal motif de la conversion est donc la transmission. Mais cette transmission doit être ici précisée. Il ne s'agit pas nécessairement de transmettre des contenus identitaires, la conversion n'étant pas nécessaire pour cela et la transmission des contenus relevant par ailleurs historiquement du père. Il s'agit bel et bien de transmettre un statut: de transmettre la judéité (comprise ici comme une identité ou comme une ethnicité) plutôt que le judaïsme (compris comme un système religieux). Ce qui explique d'ailleurs pourquoi ce sont très majoritairement les femmes qui se convertissent: elles seules transmettent la judéité. La conversion peut alors se comprendre comme un don fait à l'époux, mais peut-être encore plus aux enfants, en réponse au sacrifice que constitue pour le conjoint juif la transgression de la règle d'endogamie (Tank-Storper 2007). Elle permet de rétablir la continuité de la filiation, de réparer le chainon de la lignée que le mariage mixte avait brisé.

La conversion, dans ce contexte, n'est pas un acte symbolique mais un acte juridique. Elle prend sens dans son processus d'institutionnalisation, processus que vient sanctionner non pas tant le rituel de conversion en lui-même (qui consiste en l'immersion dans le mikvé, le bain rituel et en la circoncision pour les hommes), que l'attribution d'un certificat de conversion. Ce certificat remplace pour le converti la ketouba des parents, l'acte de mariage, qui authentifie la judéité des individus auprès des institutions à l'occasion d'un mariage à la synagogue, d'un enterrement dans un carré juif ou auprès de l'État d'Israël en cas par exemple de demande d'obtention de la citoyenneté israélienne dans le cadre de la loi du retour. Comme nous allons le voir, la validité de certificat est donc déterminante pour les candidats à la conversion, qui privilégient ainsi la légitimité de l'institution auprès de qui ils s'adressent à leur affinité idéologique ou religieuse vis-àvis de cette institution. 
Solange, qui naît en 1949, est originaire d'une très vieille famille de l'Est de la France: "À ce niveau-là nous n'avons pas de problèmes d'origine. La question ne se pose même pas. On est là, on est là...» Son père, «un homme de gauche très virulent », est maréchal-ferrant. Pendant la guerre, il est fait prisonnier et travaille à la réparation des sous-marins allemands dans une ferronnerie de Hambourg. «Dès qu'il y avait un bombardement, dès qu'il y avait quelque chose, ils avaient les mitraillettes sur la tempe pour qu'on les élimine dès qu'il y avait quoi que ce soit. Ils étaient dans un état de sousalimentation avancée puisque quand ils ont été libérés par les Américains mon père faisait 38 kilos.»

Le père de Solange revient donc très traumatisé de sa captivité en Allemagne, «mentalement perturbé », dit sa fille. Il ne parle que de la guerre, ce qui aura comme conséquence de la sensibiliser «aux problèmes de la différence et de l'horreur que peuvent apporter les guerres».

Elle quitte la maison de ses parents pour faire ses études. À X tout d'abord, à $\mathrm{Y}$ ensuite, puis revient enfin à $\mathrm{Z}$ pour suivre les cours de la faculté de médecine.

Là, elle rencontre deux jeunes étudiants juifs pratiquants à qui elle fait bientôt la cuisine. Elle apprend les règles de la casherout, puis d'autres éléments du judaïsme. Des relations privilégiées se nouent entre Solange et Serge, l'un des deux jeunes juifs, et elle envisage alors de se convertir: "À partir du moment où l'on a ce genre de relation avec quelqu'un, on doit faire ça. C'est un équilibre indispensable, c'est une question de respect pour l'autre. » Serge est très pratiquant. Il ne peut envisager de se marier avec une non-juive. Solange fait donc appel à la communauté de $\mathrm{Z}$ qui la rejette sans surprise, mais elle trouve de l'aide auprès d'un vieux "sage » qui accepte de la prendre en charge. Tous les dimanches matins, elle se rend chez lui et, durant deux heures, ils étudient aussi bien le judaïsme que la littérature française et allemande. Il encourage le couple à passer outre la «loi des hommes» et leur donne sa bénédiction pour se marier et avoir des enfants.

Ils passent donc devant le maire et Solange tombe enceinte. C'est à ce moment-là que, sous l'impulsion de ce "vieux sage ", le processus de conversion débute officiellement. Solange suit les cours de conversion de Madame Gugenheim au séminaire de la rue Vauquelin à Paris; Paris où le couple est venu s'installer afin que Serge finisse sa thèse et que Solange passe sa spécialisation de biologie humaine.

Solange est déjà pratiquante. Madame Gugenheim s'aperçoit vite de son niveau et la propose au bout d'un peu plus d'un an à la conversion. "Quand 
je me suis convertie, je connaissais depuis cinq ans la partie pratique. Mais il me manquait la partie essentielle: le fond.»

La conversion a lieu au printemps 1978. Leur fils a alors trois mois et est considéré comme juif ${ }^{4}$.

\section{Communauté d'origine et de destin $v$ s. Communauté de croyances et de pratiques}

Les règles codifiant le giyyur (le processus par lequel un non juif est fait juif, que nous traduisons ici par conversion au judaïsme) sont peu nombreuses. En substance, le Shoulkhan Aroukh ${ }^{5}$ stipule que le rituel de conversion (immersion dans le mikveh ${ }^{6}$ plus circoncision pour les hommes) est un acte irrévocable qui, s'il est mené dans les formes, ne peut être contesté 7 . Le candidat admis au rituel doit être motivé par une "intention pure» («le shem shamaïm») et doit s'engager à respecter les mitsvot (kabalat ol mitsvot). Ces clauses restrictives s'accompagnent par ailleurs du devoir, pour les rabbins, d'accepter un candidat sincère.

La notion d'intention pure place le motif de la sincérité de la démarche de conversion au centre du processus. Les motivations du candidat doivent être pures, mais - et nous le verrons, c'est très important — la pureté de l'intention n'est pas définie positivement. Rien, dans les textes de la tradition, ne vient expliciter ce qu'est être «le shem shamaim». Cela est défini négativement, par ce que ce n'est pas: vouloir se convertir pour obtenir un bénéfice ultérieur, c'est-à-dire pour de l'argent, pour accroître son pouvoir et, surtout, pour obtenir un conjoint (Sagi et Zohar 1997).

Ce dernier point pose problème quand, nous l'avons vu, entre 80 et $90 \%$ des demandes de conversion se font dans le cadre d'un mariage. En toute logique, les rabbins devraient repousser ces candidats «intéressés » sans autre examen. D'autant plus que la question des conversions dans le cadre d'un mariage pose la question de la réponse institutionnelle à la transgression religieuse. Le mariage mixte signe en effet la transgression

4. Entretien mené par l'auteur en mars 1995.

5. En hébreu "la table dressée ", code de la loi talmudique établi par Joseph Karo et publié à Venise en 1565. Il fait aujourd'hui autorité pour le judaïsme dit orthodoxe.

6. Bain rituel.

7. Maïmonide écrit ainsi dans le Mishneb Torah: «Si un converti, dont on n'aurait pas vérifié la motivation, a été circoncis et a fait l'immersion du bain rituel, quand bien même nous saurions que c'est dans un certain intérêt [autre que celui de la conversion elle-même], il sera considéré comme ayant le statut de converti.» 
d'un des principaux interdits du judaïsme: la règle d'endogamie. Et convertir l'épouse non juive d'un mariage mixte pourrait ainsi signifier la validation a posteriori de la transgression de cet interdit. Ce que refusent les institutions orthodoxes. Le motif de l'intention pure, dans ces conditions, permet de légitimer une posture plus politique de lutte contre les mariages mixtes et permet de rappeler aux époux déviants - mais plus largement à l'ensemble de la communauté - qu'il faut se marier au sein du groupe.

Les rabbins orthodoxes refusent par ailleurs de reconnaître la validité des conversions réalisées par les rabbins non orthodoxes comme les rabbins libéraux ${ }^{8}$ ou massorti ${ }^{9}$, qui de leur côté sont plus accueillants envers les conversions dans le cadre d'un mariage. La Rabbanout israélienne, l'institution religieuse orthodoxe officielle de l'État israélien dont dépend le droit personnel des citoyens israéliens de nationalité juive (un citoyen israélien peut être de nationalité juive, arabe ou druze par exemple, ce qui détermine le régime de droit personnel auquel il est assujetti et notamment le régime de son état civil), a ainsi obtenu le monopole juridique sur les conversions réalisées en Israël et tente régulièrement de l'obtenir pour toute conversion réalisée en diaspora (Klein 1997). La Rabbanout cherche ainsi

8. Le judaïsme libéral, qui est aujourd'hui majoritaire aux États-Unis mais très marginal en Israël ou en France, est né en Allemagne au XIX ${ }^{\mathrm{e}}$ siècle de la volonté d'adapter la tradition juive aux contraintes de la modernité. Cela s'est traduit par un certain nombre de réinterprétations juridiques et religieuses portant sur des points aussi variés que les règles alimentaires (qui sont euphémisées), la transmission de l'identité juive (reconnaissance de la transmission paternelle), la reconnaissance de l'égalité entre hommes et femmes et l'ordination de femmes rabbins, et, plus largement, la reconnaissance de la primauté des choix individuels sur l'héritage et sur les obligations religieuses. Dans cette logique, certaines tendances du judaïsme libéral nordaméricain militent pour la mise en place de programmes proactifs visant à recruter des convertis pour compenser le nombre des juifs de naissance qui s'éloignent du judaïsme.

9. Le terme massorti signifie littéralement «traditionnel». Le mouvement Massorti, connu aux États-Unis et au Canada sous le nom de Conservative Judaism, est l'un des trois principaux courants du judaïsme contemporain. Il se distingue d'un côté des libéraux par sa moindre acceptation des innovations juridiques et religieuses et d'un autre côté des orthodoxes par sa plus grande disposition au compromis vis-à-vis du monde contemporain. Le judaïsme massorti est ainsi plus ouvert aux demandes d'égalité entre hommes et femmes (des femmes peuvent y être ordonnées rabbin) ou aux questions liées à l'homosexualité. Ils sont favorables aux conversions dans le cadre d'un mariage, qu'ils considèrent comme une chance pour l'avenir du judaïsme, mais se déclarent hostiles à la reconnaissance automatique de la judéité des enfants nés d'un père juif et d'une mère non juive et à tout ce qui pourrait être assimilé à du prosélytisme. 
à se constituer en institution de référence du judaïsme mondial et en garante des frontières de l'identité juive, en Israël comme en diaspora.

Le monopole de la Rabbanout sur les conversions et plus largement sur la définition de la judéité ne va pas sans tensions, quand par exemple l'État d'Israël accorde la nationalité juive à des individus que la Rabbanout ne reconnaît pas comme juifs. Israël compterait aujourd'hui en effet aux alentours de 300000 citoyens qui se définissent comme juifs, qui parlent hébreu, dont les enfants servent dans l'armée israélienne, mais qui ne sont pas considérés comme juifs par le rabbinat israélien. Certains sont arrivés en Israël dans les années 1990 depuis l'ancien empire soviétique au titre de la loi du retour, d'autres sont nés en Israël. Parmi les 800000 juifs environ venus d'ex-Union soviétique durant les années 1990, près d'un tiers ne sont pas reconnus comme juifs par la Rabbanout. Ce sont soit les conjoints non juifs de ces juifs d'Union soviétique, soit les enfants de ces couples mixtes dont la mère n'est pas juive, soit encore les enfants non juifs de couples mixtes formés il y a déjà une ou deux générations en Union soviétique et qui bénéficient de la loi du retour dès lors qu'un seul de leurs grandsparents est juif. Ils ont le droit de vote à l'instar de tout citoyen israélien, mais ne peuvent se marier en Israël faute d'un mariage civil (Klein 1997, 229-294), ce qui ne les empêche cependant pas de faire des enfants qui, à leur tour, ne seront pas considérés comme juifs par les institutions du pays. Mais dans le même temps, la Rabbanout ne cède pas sur ses prérogatives juridiques qui lui attribuent le monopole sur le droit des personnes (et notamment sur le mariage et les divorces) en refusant la création d'un État civil laïc, tout en fermant la porte des conversions à ces citoyens israéliens de non-droit qui ne rentrent pas dans la définition que donne l'institution de l'identité juive. La Rabbanout a par exemple annulé en 2008 la conversion de nombreux jeunes israéliens réalisée par le rabbinat de l'armée (une institution de l'État pourtant orthodoxe) lors de leur service militaire, au prétexte que les critères d'admission n'étaient pas assez stricts (Fisher 2013, 231-236). Ce type de stratégie visant à invalider les conversions pratiquées par les institutions non orthodoxes ou pas assez orthodoxes n'est pas propre à la Rabbanout israélienne. Elle se retrouve par exemple à moindre échelle en Argentine à propos de l'attribution des places dans les cimetières juifs du pays. La AMIA, la principale institution juive ashkénaze du pays dont dépend la gestion de la plupart des cimetières juifs et qui est gérée depuis 2008 par une coalition orthodoxe, refuse désormais d'enterrer ceux qui ont été convertis par les institutions non orthodoxes et alors même que ces convertis sont par ailleurs membres de l'institution, au 
nom d'un décret de 1927 prohibant les conversions sur le sol argentin ${ }^{10}$. Des époux se voient ainsi refuser des sépultures communes quand bien même le conjoint converti n'a cessé de vivre comme un juif, de respecter rigoureusement les commandements, d'élever ses enfants comme des juifs, de fréquenter les institutions communautaires, etc., au motif qu'il n'a pas été converti au bon endroit ni par les bons rabbins.

Comment comprendre une politique qui a pour principale conséquence de troubler les identités familiales et de brouiller les frontières de la collectivité juive en faisant cohabiter des individus aux statuts hétérogènes? Force est de constater que ce type de politique n'a pour ainsi dire aucune incidence sur le nombre de mariages mixtes.

L'hypothèse que je formule est que l'intention des promoteurs de ce type de politique n'est ni de réellement lutter contre les mariages mixtes (en Argentine par exemple, malgré l'adoption du décret de prohibition évoqué supra, le nombre de mariages exogames se situe toujours autour de $50 \%$ des mariages engageant au moins un conjoint juif; Mirelman 1988, 161; Feierstein 2007, 420), ni même de réparer la confusion créée par la multiplication des mariages mixtes en homogénéisant les statuts religieux des conjoints, mais de défendre une conception de la collectivité fondée sur l'observation d'un certain nombre de principes intangibles. Plutôt que la judéité (l'idée d'une identité juive conçue comme une communauté d'origine et de destin), les rabbins orthodoxes entendent défendre le judaïsme: l'idée d'une identité juive fondée sur un ensemble de croyances et de pratiques partagées. Les rabbins sacrifient ainsi la cohérence communautaire et familiale au nom de l'intégrité du message que la collectivité est chargée de porter.

10. En 1927, le rabbin d'origine syrienne, Shaul David Setton, en charge de la communauté Hesed Schel Emeth Sefaradit de Buenos Aires, avec l'appui du rabbin ashkenaze de Moisesville Aharon Halevi Goldman et avec l'approbation des grands rabbins séfarades et ashkenazes de Palestine, édicta la prohibition totale et définitive de toute conversion à l'intérieur des frontières de la République argentine. L'objectif explicite de ce décret (qui ne connaît pas d'équivalent) était de lutter contre la multiplication des mariages exogames et de mettre un terme à des politiques de conversion jugées trop laxistes et susceptibles de mettre en danger l'intégrité et la pérennité de l'identité juive en Argentine. Si l'application de ce décret n'eut pour ainsi dire aucune influence sur le nombre des mariages mixtes, il a en revanche permis la progressive homogénéisation religieuse des communautés juives argentines, jusque-là relativement atomisées (Tank-Storper 2005). Dans les faits, le décret de prohibition permet aux rabbins orthodoxes d'invalider toute conversion réalisée sur le sol argentin sans autre examen. 
Il s'agit, en d'autres mots, d'affirmer que le fondement de l'identité juive réside en premier lieu dans un corpus de croyances, mais surtout de pratiques, et secondairement dans une origine commune ou un destin national. À ce titre, les candidats qui remplissent les conditions posées par les institutions (c'est-à-dire qui démontrent leur conformité avec les attentes des rabbins et des institutions) sont acceptés à la conversion quelle que soit leur situation matrimoniale. De même, les enfants de père juif et de mère non juive non convertie ne sont pas reconnus comme juifs quand bien même ils s'identifient comme tels et qu'ils fréquentent les activités communautaires (y compris les activités des communautés orthodoxes). Il leur reviendra alors de se convertir le moment venu, mais au prix d'une mise en conformité avec les exigences des rabbins.

C'est en d'autres termes une logique cléricale qui sous-tend les positions orthodoxes en matière de conversion. Il s'agit d'imposer la définition d'une collectivité juive conçue comme communauté de croyances et de pratiques (pour être plus précis, l'idée d'une collectivité juive conçue comme une communauté de pratiquants soumis à la loi), unie autour d'institutions garantes de l'intégrité et de la pérennité des contenus de l'identité. Dès lors, c'est le maintien de la cohérence religieuse qui constitue le principal enjeu normatif et, à l'inverse du groupe ethnique pour qui, si l'on s'en réfère à Barth, la définition des contenus de l'identité est au service de la préservation et de la clarification des frontières du groupe (Barth 1995), les frontières de l'identité sont ici soumises aux contenus de la croyance.

\section{Convertir}

Il s'agit donc, pour les rabbins orthodoxes, de défendre à travers la politique de conversion mise en œuvre, l'idée d'une collectivité juive définie par sa conformité avec les normes religieuses des institutions. Mais en quoi consistent précisément ces normes? Pour le judaïsme rabbinique, la bonne religiosité réside non pas tant dans l'adhésion à des énoncés de croyance que dans la mise en œuvre des commandements et dans la soumission aux autorités que sont les rabbins. S'interrogeant sur la notion d'hérésie (et par là-même de la norme) dans le judaïsme rabbinique, Goldish note ainsi:

La plupart [des passages issus de la littérature rabbinique traitant des hérétiques] laissent entendre que le péché imputable à ces personnes ne tient pas tant à leurs convictions à propos de Dieu qu'à leur transgression des commandements enjoignant d'honorer et de se soumettre à la volonté des rabbins (l'enseignement sur la résurrection, par exemple) ou 
à leur responsabilité dans les dissensions entre les communautés. (Goldish 2012, 257)

Il s'agit en d'autres mots davantage d'une orthopraxie que d'une orthodoxie (Azria, 1996); d'un croire en acte (Hervieu-Léger 1993). Le bon juif n'est pas celui qui fait profession de foi, mais celui qui se conforme aux commandements et qui se soumet à l'autorité des docteurs de la loi que sont les rabbins.

Quelles sont les conséquences pour les candidats qui se convertissent auprès des institutions orthodoxes, concernant notamment la manière dont ils conçoivent et élaborent leur propre judéité et les pratiques qui l'accompagnent? Si, comme je l'ai déjà signalé, les candidats à la conversion n'investissent au début de leur processus que marginalement les dimensions religieuses de l'identité juive, si, en d'autres mots, ils se tournent vers un judaïsme perçu plus comme une éthique, une culture ou une ethnicité, que comme une religion, c'est cependant presque exclusivement les dimensions religieuses qu'ils devront apprendre et mettre en pratique pour obtenir la conversion auprès de la Rabbanout israélienne ou d'une institution comme le Consistoire central de France, de sensibilité proche de la Rabbanout israélienne. Ce qui ne va pas sans conflit.

Pour la plupart des candidats, la pratique du judaïsme se limite au début de leur processus de conversion au respect des grandes fêtes (Kippour, Pessah, Soukhot) et à quelques gestes symboliques (allumage des bougies de shabbat). Une enquêtée, parlant de ses pratiques au moment de son premier rendez-vous avec le rabbin du bureau des conversions du Consistoire central de France, me dit ainsi: «Par rapport à la casherout, ça faisait longtemps que je ne mangeais plus de porc, peu de viande, mais je n'allais pas dans les boucheries casher tout simplement parce que je n'osais pas y aller. C'était tout. Je ne pratiquais pas énormément. » Le respect de l'interdit du porc semble être le premier geste que s'autorisent ces candidats. Il vient marquer une première rupture qui symbolise la séparation et l'engagement. Pour d'autres, il peut s'agir de quelques pratiques symboliques qui permettent de s'inscrire dans une "spiritualité " (allumage des bougies de shabbat, kiddoush occasionnel, etc.).

Les pratiques des candidats à la conversion entrent cependant en contradiction avec les exigences posées par les rabbins orthodoxes. Une candidate raconte ainsi son premier entretien avec le rabbin en charge du «bureau des conversions» au Consistoire Central de France: 
[...] il y a eu, lors du premier entretien [avec le rabbin du Consistoire], un petit échange vif sur sa manière d'envisager les règles religieuses qu'il présentait volontairement comme un mécanisme. Je lui disais «tout à fait, mais je veux aussi faire une recherche de sens ". Il m'a répondu que je devais m'habituer à être une petite juive: "parce que le matin, à Shabbat, quand vous vous réveillez, il faut penser à ne pas allumer la lumière, etc. ».

Pour les candidats, les gestes sont avant tout mobilisés comme des marqueurs d'appartenance, comme un répertoire au sein duquel puiser pour signifier l'identité et le sens. Ils sont un support à l'expérience religieuse et identitaire. Pour les rabbins orthodoxes, au contraire, mettre en pratique les gestes rituels, respecter la Loi, c'est s'inscrire dans une sujétion absolue à l'autorité du Texte et de ses intermédiaires, les rabbins. La pratique n'est pas une superstructure de l'identité. Elle n'est pas non plus expérimentation. Elle trouve sa fin en elle-même. La casherout, le respect du Shabbat, la prière, etc., ne sont pas des moyens pour s'élever spirituellement et encore moins, comme certaines interprétations modernistes voudraient parfois le penser, des moyens pour manger plus sainement ou pour assurer la bonne cohésion sociale (Hansel 1998, 34). La pratique des mitsvot est la condition de l'identité, pas le signe.

L'enjeu du processus de conversion sera donc pour les candidats de se fondre dans la conception orthodoxe de la pratique des commandements, de passer d'une pratique en grande partie symbolique et "authentique", d'une pratique expérientielle, à une pratique plus systématique, conforme, normée. La conversion, dans ce contexte, n'est pas tant une conversion de l'âme, ni même une conversion à une autre croyance, mais une conversion à un autre rapport à la pratique religieuse, qui relègue la quête de sens au second plan pour se concentrer sur le geste et la corporalité, mais surtout sur l'obligation.

En somme, pour les candidats comme pour les rabbins, tout s'efface derrière l'attestation factuelle de la sincérité, incarnée par la mise en pratique inconditionnelle des mitsvot. Progressivement, la preuve de la sincérité prend le pas sur la sincérité elle-même, pourtant énoncée par l'institution à travers l'obligation du le shem shamaim comme critère ultime de la valeur d'une démarche de conversion. Placé au centre du dispositif, le le shem shamaim, l'intention pure, se mue ainsi en une démonstration pratique. Mais, ce faisant, la sincérité est dissoute au profit de la formalité et de la performance. Si les candidats deviennent formellement sincères, s'ils donnent des preuves formelles de leur sincérité, cela se fait au détriment de ce qu'ils jugent être la sincérité légitime, c'est-à-dire au 
détriment de l'authenticité de leur démarche. Et c'est bien quand les candidats se fondent dans la conception rabbinique de la pratique qu'ils sont admis à la conversion. Ce que démontre notamment l'attitude des rabbins envers les conversions par mariage. Car en dépit des motifs apparents d'intéressement, les rabbins orthodoxes ne s'interdisent pas de convertir les époux non juifs d'un mariage mixte. À condition toutefois que les candidats (le plus souvent les candidates), mais aussi les conjoints et les éventuels enfants se "convertissent" également aux pratiques juives orthodoxes. Conversion qui ne va pas nécessairement de soi dans la mesure où les conjoints, déjà juifs, ne sont pas toujours disposés à se voir imposer par l'institution une conception de l'identité juive qu'ils ne partagent pas nécessairement.

Ce que les candidats à la conversion en demande de validation institutionnelle sont prêts à accepter comme une contrainte plus ou moins légitime (ils acceptent de se plier aux exigences de l'institution en échange de la conversion, quitte à renégocier leur degré de pratique avec leur conjoint une fois la conversion obtenue) pose en effet beaucoup plus de problèmes à ces conjoints qui revendiquent le droit d'être juifs à leur manière, sans avoir à en rendre compte aux autorités religieuses. Le cas de cette jeune femme d'origine suisse qui entreprend de se convertir après s'être installée en Israël et y avoir rencontré son mari avec qui elle a deux enfants est de ce point de vue très parlant. Son mari est arrivé très jeune en Israël. Ni son israélité ni sa judéité ne lui semblent devoir être démontrées ni justifiées. Le fait que sa femme ne soit pas juive au sens qu'en donne la Halakha (la loi juive) ne lui pose par ailleurs pas plus de problème, si bien que sa conversion ne lui apparaissait pas nécessaire. Or, la première question qui fut posée à la jeune femme lors de son premier contact avec la Rabbanout israélienne fut celle de la profession de son mari. Ce dernier travaillait durant Shabbat. La réponse des rabbins fut sans appel: "Le jour où votre mari vivra comme un vrai juif, revenez nous voir et nous verrons ce que l'on peut faire pour vous.»

La réussite du processus de conversion de cette femme était ainsi directement liée aux pratiques de son époux. Quoi qu'elle puisse faire, elle ne sera jamais admise au giyyur si son mari ne rentre pas, lui non plus, dans une démarche de conversion à une autre conception de la judéité, centrée autour des pratiques religieuses. Ce qu'il refuse. Lors d'un entretien que j'eus avec lui, il me dit: "Qui sont-ils pour me dire ce que je dois faire?" Et à l'heure actuelle, la jeune femme n'est toujours pas convertie et ses enfants ne sont pas juifs. Ils n'en sont pas moins Israéliens. Ils parlent 
hébreu, feront l'armée, vivront probablement en Israël, mais ne pourront pas s'y marier...

La situation est encore plus problématique dans le cas de la conversion d'enfants adoptés. Même si la mère adoptive est juive, l'enfant adopté dont on ne connaît pas l'origine doit être converti pour être considéré comme juif aux yeux des institutions orthodoxes. Les jeunes parents, le plus souvent, s'attendent à ce que la conversion de leur enfant soit une formalité: leur identité juive ne faisant aucun doute, celle de leur enfant leur semble aller de soi. Et pourtant, les rabbins n'accordent la conversion qu'à la condition exclusive que les deux parents fassent «t'chouva», c'est-à-dire deviennent des juifs pratiquants et s'engagent à élever leur enfant dans le strict respect des mitsvot. Ce qu'ils vivent comme une remise en cause de leur propre judéité (Nizard 2012).

Le cas de ces conversions d'enfants adoptés est d'autant plus intéressant qu'il me semble mettre le doigt sur cette tension qui oppose deux conceptions de l'identité juive. Pour ces parents adoptifs qui ne sont pas nécessairement religieux mais dont la judéité va de soi, l'identité juive est avant tout une filiation qui passe par le sang ou par le cœur (Nizard 2012, 187). Puisqu'ils sont juifs, leur enfant est juif; son identité ne peut dépendre de la validation d'une institution, d'autant moins quand cette institution conditionne cette validation à la mise en acte d'une judéité qui va à l'encontre de leurs propres conceptions de l'identité. Pour les rabbins, au contraire, l'identité juive est un bien commun qui doit être acté juridiquement selon les principes de la Torah, eux-mêmes confiés aux soins des rabbins. Elle n'est pas identification (individuelle et subjective) mais identité (formelle et institutionnalisée). Et cette identité passe par la mise en conformité avec les principes normatifs des institutions qui s'estiment en charge de son intégrité et de sa pérennité.

On le voit, les politiques orthodoxes de conversion sont mises au service d'une politique plus large de «rejudaïsation» des populations juives. En affirmant que devenir juif, c'est devenir un juif pratiquant et non un juif ethnique ou un juif national, les institutions entendent, si ce n'est imposer, pour le moins témoigner voire sauvegarder l'idée qu'elles se font de ce qu'être juif veut dire: un collectif soumis à la loi. Elles entendent lutter contre ce qu'elles considèrent être une réification de l'identité juive dans une entité nationale israélienne ou contre sa réduction à un groupe ethnique ou à une identité dont l'héritage ne constituerait plus un principe structurant mais se réduirait à quelques traits discriminants. 


\section{Références}

Allouche-Benayoun, J. (1997), "Conversion au judaïsme et enjeux de mémoire. Une affaire de femmes? ", dans F. Lautman, dir., Ni Eve ni Marie. Luttes et incertitudes des héritières de la Bible, Genève, Labor et Fidès.

Azria, R. (1996), "Le Croire dans le judaïsme », dans L. BABÈs, dir., Les Nouvelles manières de croire, Paris, L’Atelier, p. 93-108.

BARTH, F. (1995), Les groupes ethniqes et leurs frontières, dans P. PouTIGNAT et J. Streiff-Fenart, Théories de l'ethnicité, Paris, PUF, p. 203-249.

Feierstein, R. (2007), Vida cotidiana de los Judios argentinos. Del gueto al country, Buenos Aires, Editorial Sudamericana.

Fisher, N. (2013), "A Jewish State? Controversial Conversions and the Dispute Over Israel's Jewish Character ", Contemporary Jewry, 33/3, p. 217-240.

Barack Fishman, S. (1999), "The Changing American Jewish Family Faces the 1990s ", dans R. Rosenberg Farber et C. I. Waxman, dir., Jews in America. A contemporary Reader, Brandeis University Press, Hanover and London.

Forster, B. et Taвachnik, J. (1991), Jews by Choice. A Study of converts to Reform and Conservative Judaism, Hoboken, KTAV.

Goldish, M. (2012), "Y a-t-il des hérésies juives? ", dans J. BAumgarten et J. Darmon, dir., Aux origines du judaïsme, Paris/Arles, Les liens qui libèrent/Actes Sud, p. 254-273.

Hansel, G. (1998), Explorations talmudiques, Paris, Odile Jacob.

Hervieu-Léger, D. (1993), La Religion pour mémoire, Paris, Cerf.

Klein, C. (1997), La démocratie d'Israël, Paris, Le Seuil.

Levin, M. (1995), Segunda investigacion sobre las conversiones en el Seminario Rabinico Latinoamericano, Instituto de Introduccion al Judaismo, Seminario Rabínico Latinoamericano.

Mayer, E. (1977), "Jews by Choice. Their Impact on the Contemporary American Jewish Community ", dans M. Fine and M. Himmelfarb, dir., American Jewish Year Book, New York/Philadelphia, American Jewish Committee/Jewish Publication Society.

Mirelman, V. A. (1988), En Busceda de una identidad. Los inmigrantes judios en Buenos Aires 1890-1930, Buenos Aires, Mila. 
Montgomery, S. E. (1991), The Integration of Jews by Choice into the Philadelphia Jewish Community, Dissertation doctorale présentée au Graduate School-New Brunswick Rutgers, The State University of New Jersey.

Nizard, S. (2012), Adopter et transmettre. Filiations adoptives dans le judaïsme contemporain, Paris, Éditions EHESS.

Poutignat, P. et Streiff-Fenart, J. (1995), Théories de l'ethnicité, Paris, PUF.

Ricceur, P. (1990), Soi-même comme un autre, Paris, Seuil.

SAGI, A. et Zohar, Z. (1997), "Giyyur, identité juive et modernisation: une analyse des sources halakhiques ", dans F. Heymann et D. STORPER Perez, dir., Le corps du texte. Pour une anthropologie des textes de la tradition juive, Paris, éd. du CNRS, p. 293-318.

Stern, Y. Z. (2013), Conversion in Israël. From Bethlehem to Chemm to Sodom, The Israel Democracy Institute, disponible sur <http://en.idi. org.il/analysis/articles/conversion-in-israel-from-bethlehem-to-chelmto-sodom>.

TANK-STORPER, S. (2005), «La conversion prohibée. Mariages mixtes et politique de conversion dans le champ religieux juif argentin ", Archives de Sciences Sociales des Religions, 131-132, p. 123-142.

\section{Éditions.} (2007), Juifs d'élection. Se convertir au judaïsme, Paris, CNRS

Weber, M. (1996), Sociologie des religions, Paris, Gallimard.

\section{Résumé}

En affirmant que devenir juif, c'est devenir un juif pratiquant et non un juif «ethnique» ou «national», et en tentant de s'arroger le monopole sur les conversions au judaïsme, notamment en Israël, les institutions religieuses de sensibilité orthodoxe défendent l'idée qu'elles se font de ce qu'être juif veut dire: un collectif soumis à la loi. Elles entendent ainsi lutter contre ce qu'elles considèrent être une réification de l'identité juive dans une entité nationale israélienne ou contre sa réduction à un groupe ethnique ou à une identité dont l'héritage ne constituerait plus un principe structurant mais se réduirait à quelques traits discriminants. 


\section{Abstract}

By claiming that becoming Jewish is becoming a religious Jew rather than an ethnic or national Jew, and by trying to impose this idea by the way of keeping the monopoly on conversions to Judaism in Israel and the diaspora, orthodox religious institutions stand up for their idea of collective Jewishness -meaning a collective submitted to the Jewish law. Their purpose is also to fight against what they understand as a reification of Jewish identity in a national or an ethnic identity which heritage wouldn't be a structuring principle anymore, but a would be reduced to some discriminants features. 